\title{
Supporting Information: Stretching a Semiflexible Polymer of Finite Length
}

\author{
Nigel T. Andersen, Yue Teng, and Jeff Z. Y. Chen* \\ Department of Physics and Astronomy, University of Waterloo, Ontario, Canada, N2L $3 G 1$ \\ E-mail: jeffchen@uwaterloo.ca
}

\section{Green's Function Theory}

To calculate the statistical properties for the stretched wormlike chain (WLC), we use the Green's function approach. The Green's function $G\left(\mathbf{r}_{\mathbf{0}}, \mathbf{u}_{\mathbf{0}} ; \mathbf{r}, \mathbf{u} ; t\right)$ represents the partition function of a chain of length $L t$, with the initial point labeled by $t=0$ at position $\mathbf{r}_{\mathbf{0}}$ pointing in the direction $\mathbf{u}_{\mathbf{0}}$, and end labeled by $t=1$, at position $\mathbf{r}$ pointing in the direction $\mathbf{u}$. At $t=0$, the Green's function takes the value

$$
G\left(\mathbf{r}_{\mathbf{0}}, \mathbf{u}_{\mathbf{0}} ; \mathbf{r}, \mathbf{u} ; t=0\right)=\delta\left(\mathbf{r}_{\mathbf{0}}-\mathbf{r}\right) \delta\left(\mathbf{u}_{\mathbf{0}}-\mathbf{u}\right) .
$$

A reduced Green's function, or propagator, $q(\mathbf{r}, \mathbf{u}, t)$ is obtained by integrating over the coordinate set associated with the $t=0$ terminal end,

$$
q(\mathbf{r}, \mathbf{u} ; t)=\int \mathrm{d} \mathbf{r}_{0} \mathrm{~d} \mathbf{u}_{0} G\left(\mathbf{r}_{\mathbf{0}}, \mathbf{u}_{\mathbf{0}} ; \mathbf{r}, \mathbf{u} ; t=0\right),
$$

and represents the partition function for all configurations with the coordinate set of the $t$ terminal specified. The total partition function for all configurations is then calculated 
through

$$
Q=\int \operatorname{dud} \mathbf{r} q(\mathbf{u}, t=1)
$$

for the entire chain when $t=1$.

The general Hamiltonian for a WLC under an external field $U$ is

$$
\beta H=\int_{0}^{1} \mathrm{~d} t\left[\frac{\lambda}{2 L}\left|\frac{\mathrm{d} \mathbf{u}(t)}{\mathrm{d} t}\right|^{2}-U[\mathbf{r}(t), \mathbf{u}(t)]\right] .
$$

By building the polymer through the addition of infinitesimal segments of length $L \mathrm{~d} t$, the propagator for such a Hamiltonian can be shown to satisfy the diffusion-like partial differential equation,,$\underline{1}$

$$
\frac{\partial}{\partial t} q(\mathbf{r}, \mathbf{u}, t)=\left[-L \mathbf{u} \cdot \nabla_{\mathbf{r}}+\frac{L}{2 \lambda} \nabla_{\mathbf{u}}^{2}-U(\mathbf{r}, \mathbf{u})\right] q(\mathbf{r}, \mathbf{u}, t) .
$$

For a free-space WLC stretched along the $z$ direction by force $\mathbf{f}=f \hat{z}$, the propagator has both translational and azimuthal symmetry, making it a function of the angle from the $z$-axis $\theta$ and $t$ only. Spherical coordinates is a natural choice to represent this symmetry. More specifically,

$$
\frac{\partial}{\partial t} q(\theta, t)=\left[\frac{L}{2 \lambda}\left(\frac{1}{\sin \theta} \frac{\partial}{\partial \theta} \sin \theta \frac{\partial}{\partial \theta}\right)+\beta L f \cos \theta\right] q(\theta, t)
$$

as given in the main text.

\section{Strongly stretched regime and the Feynman integral}

Under the strongly stretched condition, the wormlike chain Hamiltonian can be expanded to have the quadratic form $\underline{2}$

$$
\beta H=\int_{0}^{1} \mathrm{~d} t\left[\frac{\lambda}{2 L}\left|\frac{\mathrm{d} \mathbf{u}_{x y}(t)}{\mathrm{d} t}\right|^{2}+\frac{1}{2} \beta L f \mathbf{u}_{x y}^{2}(t)\right]-\beta L f .
$$


The projection of $\mathbf{u}$ on the $x y$-plane, $\mathbf{u}_{x y}$, is a two dimensional vector. Marko and Siggia used this approximation for the limit $\tilde{L} \gg 1$, and Kessler and Rabin as well as Kierfeld et al. for all $\tilde{L}$, functioning as the starting point of the theoretical studies on strongly stretched chains.

A critical step is to map the above problem to the quantum-mechanical problem of carrying out the Feynman path integral of a two-dimensional particle in a simple harmonic potential well. The two-point Green's function, for the probability of finding a polymer segment of length $\tau=t-t^{\prime}$ with the terminal ends labeled $t$ and $t^{\prime}$ and having $\mathbf{u}_{x y}$ and $\mathbf{u}_{x y}^{\prime}$, has the form, $\underline{\underline{5}}$

$$
G\left(\mathbf{u}_{x y}, \mathbf{u}_{x y}^{\prime} ; \tau\right)=A e^{-B\left(\mathbf{u}_{x y}^{2}+\mathbf{u}_{x y}^{\prime}{ }^{2}\right)-2 C \mathbf{u}_{x y} \cdot \mathbf{u}_{x y}^{\prime}}
$$

where

$$
\begin{aligned}
& A=\frac{\alpha}{2 \pi} \frac{1}{\sinh \gamma \tau}, \\
& B=\pi A \cosh \gamma \tau,
\end{aligned}
$$

and

$$
C=-\pi A
$$

To simplify the notation,

$$
\alpha=(\lambda \beta f)^{1 / 2} \quad \text { and } \quad \gamma=L(\beta f / \lambda)^{1 / 2}
$$

are introduced above. Based on this, one can obtain the partition function

$$
\frac{Q}{V}=\int \mathrm{d} \mathbf{u}_{x y} \mathrm{~d} \mathbf{u}_{x y}^{\prime} G\left(\mathbf{u}_{x y}, \mathbf{u}_{x y}^{\prime} ; 1\right)=\frac{2 \pi}{\alpha \sinh \gamma},
$$

which leads to

$$
\langle z\rangle / L=1-D(L \sqrt{\beta f / \lambda}) / \beta f L \quad(\text { for } \tilde{f} \gg 1 \text { and any } \tilde{L})
$$


where

$$
D(x)=[1+x \operatorname{coth}(x)] / 2 .
$$

as is presented in the main text.

The orientational correlation function, for the two monomers located at $t$ and $t^{\prime}$, can be obtained based on the correlation function, $G\left(\mathbf{u}_{0}, \mathbf{u}, \mathbf{u}^{\prime}, \mathbf{u}_{1} ; t, t^{\prime}\right) \equiv G\left(\mathbf{u}_{0}, \mathbf{u} ; t\right) G\left(\mathbf{u}, \mathbf{u}^{\prime} ; t-\right.$ $\left.t^{\prime}\right) G\left(\mathbf{u}^{\prime}, \mathbf{u}_{1} ; 1-t^{\prime}\right)$, where the $\mathbf{u}$-vectors are all two-dimensional on the $x y$-plane. Carrying out the integral, one obtains

$$
\left\langle\mathbf{u} \cdot \mathbf{u}^{\prime}\right\rangle=\frac{2 \cosh (\gamma t) \cosh \left[\gamma\left(1-t^{\prime}\right)\right]}{\alpha \sinh \gamma}, \quad \text { when } t^{\prime} \geq t
$$

The projected mean square end-to-end distance can then be evaluated

$$
\left\langle\mathbf{R}_{x y}^{2}\right\rangle=L^{2} \int_{0}^{1} \mathrm{~d} t_{1} \int_{0}^{1} \mathrm{~d} t_{2}\left\langle\mathbf{u}\left(t_{1}\right) \cdot \mathbf{u}\left(t_{2}\right)\right\rangle=\frac{2 L}{\beta f} .
$$

This rather simple result was noted by Lagowski et al., which corrected a mistake in the

review by Freed, though their work was for a three-dimensional $\mathbf{u} ; \underline{\underline{6}} \underline{\underline{7}}$ this changes the coefficient 2 in the above to their 3.

\section{The GSD theory}

In the asymptotic $\tilde{L} \gg 1$ limit, one can assume that the propagator takes a variable-separated form

$$
q(\theta, t)=\exp (-\mu \tilde{L} t) q(\theta)
$$

This is known as the ground-state dominance (GSD) theory, where the $q$-function with a single argument is used to represent the GSD dependence on $\theta$. One can then simplify the 
modified diffusion equation into

$$
-\mu q(\theta)=\left[\left(\frac{1}{\sin \theta} \frac{\partial}{\partial \theta} \sin \theta \frac{\partial}{\partial \theta}\right)+2 \lambda \beta f \cos \theta\right] q(\theta)
$$

in which only one variable, $\theta$, and one parameter, $2 \lambda \beta f$, appear. The other parameter, $\tilde{L}$, is dropped from the problem completely. Note that in the $\tilde{L} \gg 1$ limit, $\tilde{f}=2 \lambda \beta f$ can be directly used.

The partition function is then

$$
\ln Q=-\mu \tilde{L}+\mathcal{O}\left(\tilde{L}^{0}\right)
$$

and hence the extension is given by

$$
\frac{\langle z\rangle}{L}=-\frac{\partial \mu}{\partial \tilde{f}}
$$

Therefore to solve the flexible chain problem, one only needs to solve for the eigenvalue problem in (15).

Marko and Siggia solved this problem through expanding $q(\theta)$ via a Legendre series up to degree 100. We reproduced the numerical result for selected $\tilde{f}$ values, following the procedure laid out in. $\underline{\underline{2}}$ The result is then used in Fig. 2 in the main text, as the dashed curve and compared with formulae proposed by Marko and Siggia,,$\underline{2}$ Bouchiat et al.,,$\underline{\underline{8}}$ and Petrosvan,,$\underline{9}$ as illustrated in Fig. 1 (c), (d), and (e) in the main text. Note that we used a different numerical scheme (Crank-Nicolson, discussed in the main text) to solve the diffusion equation (Eq. 4 in the main text) with the full $\theta$ - and $t$-dependencies. Our large $\tilde{L}$ sets, from the full solution, completely agree with the Legendre series approach based on the GSD theory. Our procedure further justifies the numerical procedure taken in, $\underline{\underline{2}}$ and vice versa. 


\section{References}

(1) Liang, Q.; Li, J.; Zhang, P.; Chen, J. Z. Y. Modified diffusion equation for the wormlikechain statistics in curvilinear coordinates. J. Chem. Phys. 2013, 138, 244910.

(2) Marko, J. F.; Siggia, E. D. Stretching DNA. Macromolecules 1995, 28, 8759-8770.

(3) Kessler, D. A.; Rabin, Y. Distribution functions for filaments under tension. J. Chem. Phys. 2004, 121, 1155-1164.

(4) Kierfeld, J.; Niamploy, O.; Sa-Yakanit, V.; Lipowsky, R. Stretching of semiflexible polymers with elastic bonds. Eur. Phys. J. E 2004, 14, 17-34.

(5) Feynman, R. P. Statistical Mechanics: A Set Of Lectures; Westview Press, 1998.

(6) Lagowski, J. B.; Noolandi, J.; Nickel, B. Stiff chain model-functional integral approach. J. Chem. Phys. 1991, 95, 1266-1269.

(7) Freed, K. F. Advances in Chemical Physics; John Wiley \& Sons, Ltd, 1972; Vol. 22; pp $1-128$.

(8) Bouchiat, C.; Wang, M. D.; Allemand, J.-F.; Strick, T.; Block, S. M.; Croquette, V. Estimating the Persistence Length of a Worm-Like Chain Molecule from Force-Extension Measurements. Biophys. J. 1999, 76, 409-413.

(9) Petrosyan, R. Improved approximations for some polymer extension models. Rheol Acta 2017, 56, 21-26. 